\title{
The effect of physician educational intervention on venous thromboembolism pharmacological prophylaxis in medical inpatients from the respiratory department: a retrospective cohort study
}

\author{
Hanqi Wang ${ }^{1}$, Yalin Pan ${ }^{1}$, Tongtong Chen ${ }^{1}$, Liuping Chen ${ }^{1}$, Haiying Lv ${ }^{1}$, Erzhen Chen ${ }^{2}$ Jieming $\mathbf{Q u}^{3}$, \\ Wei Tang ${ }^{3}$, Wanying $\mathrm{Xu}^{4}$, Yong $\mathrm{Lu}^{1}$ \\ ${ }^{1}$ Department of Radiology, Ruijin Hospital, School of Medicine, Shanghai Jiao Tong University, Shanghai, China; ${ }^{2}$ Intensive Care Unit, Ruijin \\ Hospital, School of Medicine, Shanghai Jiao Tong University, Shanghai, China; ${ }^{3}$ Department of Respiratory, Ruijin Hospital, School of Medicine, \\ Shanghai Jiao Tong University, Shanghai, China; ${ }^{4}$ Department of Medical Affairs, Ruijin Hospital, School of Medicine, Shanghai Jiao Tong \\ University, Shanghai, China \\ Contributions: (I) Conception and design: Y Lu, E Chen; (II) Administrative support: Y Lu, J Qu; (III) Provision of study materials or patients: W \\ Tang, W Xu; (IV) Collection and assembly of data: Y Pan, T Chen, L Chen, H Lv; (V) Data analysis and interpretation: H Wang; (VI) Manuscript \\ writing: All authors; (VII) Final approval of manuscript: All authors. \\ Correspondence to: Yong Lu, MD. Department of Radiology, Ruijin Hospital, School of Medicine, Shanghai Jiao Tong University, Shanghai, China. \\ Email: 18917762053@163.com.
}

Background Venous thromboembolism (VTE) is a serious health problem for which pharmacological prophylaxis has been proven to be effective. However, there are significant gaps between the guidelines and clinical practice. This study is to evaluate the effect of physician educational intervention (PEI) on VTE pharmacological prophylaxis in medical inpatients from the respiratory department.

Methods: Medical inpatients from the respiratory department between February 2014 and December 2016 were recruited in this retrospective cohort study. They were assigned to the PEI group or the control group according to whether their physicians undertook a quality improvement (QI) project carried out in hospital to raise physician awareness of pharmacological thromboprophylaxis by educational intervention. Any and appropriate pharmacological VTE prophylaxis rates, the use of appropriate anticoagulants, and the occurrence of VTE events in the two groups were calculated and compared using a chi-square test and continuity correction. Poisson regression analysis was used to evaluate the relative risk (RR) of PEI on the occurrence of VTE events.

Results: The any pharmacological VTE prophylaxis rate $(11.3 \%$ vs. $5.9 \%, \mathrm{P}=0.048)$ and appropriate pharmacological VTE prophylaxis rate $(9.3 \%$ vs. $5.5 \%, \mathrm{P}=0.036)$ in high-risk patients without high major bleeding risk were both significantly higher than the control group. Compared with the control group, appropriate anticoagulants in the PEI group took up a larger proportion of all used anticoagulants $(90.3 \%$ vs. $78.7 \%, \mathrm{P}=0.007)$. In anticoagulants used for high-risk patients without high major bleeding, appropriate anticoagulants show no statistical difference between the two groups (93.8\% vs. $77.8 \%, \mathrm{P}=0.153)$. There was no difference in the occurrence of VTE events between the two study groups in overall patients $(0.5 \%$ vs. $0.6 \%, \mathrm{P}=0.913)$, and among those with high VTE risk $(1.7 \%$ vs. $1.0 \%, \mathrm{P}=0.554)$. PEI had no association with the probability of VTE event occurrence (RR, 1.246; 95\% CI, 0.478-2.188, P=0.954).

Conclusions: Educational intervention effectively increased physician awareness of VTE prophylaxis in the respiratory department. Further interventions are still necessary since the guidelines were implemented to a relatively low degree.

Keywords: Venous thromboembolism (VTE); thromboprophylaxis; education intervention; respiratory; medical inpatients 
Submitted Aug 26, 2020. Accepted for publication Oct 30, 2020.

doi: 10.21037/apm-20-1833

View this article at: http://dx.doi.org/10.21037/apm-20-1833

\section{Introduction}

Venous thromboembolism (VTE), including pulmonary embolism (PE) and deep vein thrombosis (DVT), has become a serious health problem worldwide, with significant morbidity and mortality (1-3). It is the third most frequent cardiovascular disease in Western countries, with an incidence of approximately 2 per 1,000 in the USA $(4,5)$. It is reported that inpatients have a 100 -fold increase in the risk of VTE (6). In respiratory department, risk factors like elderly age, lung cancer, respiratory failure and respiratory tract infections are usually common, therefore a large portion of inpatients are with high VTE risk and VTE prophylaxis are of great importance in respiratory department $(7,8)$. Moreover, relevant studies of VTE prophylaxis in respiratory department are still limited.

Pharmacological VTE prophylaxis, such as treatment with low-molecular-weight heparin (LMWH) and lowdose unfractionated heparin (UFH), has been confirmed to effectively decrease the incidence of VTE in high-risk patients (9). However, significant gaps remain between the guidelines for the use of pharmacological prophylaxis and their administration in clinical practice. This might be due to a lack of physician awareness of the clinical criteria for prophylaxis. A previous study demonstrated that in patients judged to be at risk for VTE according to the American College of Chest Physicians (ACCP) criteria, only $58.5 \%$ of surgical patients and $39.5 \%$ of medical patients received ACCP-recommended VTE prophylaxis (10). Therefore, it is necessary to improve physician awareness of the importance and standard usage of pharmacological thromboprophylaxis. Educational intervention appears to be useful, as several studies have demonstrated an increased rate of VTE prophylaxis after intervention (11-14). In this retrospective study, we aimed to evaluate the effect of a quality improvement (QI) project to raise physician awareness of pharmacological VTE prophylaxis through educational intervention on medical inpatients from the respiratory department. Results showed that this project significantly increased the appropriate VTE prophylaxis rate in high-risk medical inpatients without high major bleeding risk, as well as the use of appropriate anticoagulants. The present study provide evidence on realworld management of VTE in respiratory department and educational intervention for VTE prophylaxis in a Chinese population, with a lack of these data in previous study. We believe our results are significant for VTE management in hospital and government policy. We present the following article in accordance with the STROBE reporting checklist (available at http://dx.doi.org/10.21037/apm-20-1833).

\section{Methods}

\section{Study design and population}

We conducted a single-center retrospective cohort study extracting collected data from the Hospital Information System (HIS). The QI project was started in April 2015 in Ruijin Hospital, Shanghai Jiao Tong University School of Medicine, China. During the project, physicians learned the ninth edition of the ACCP guidelines about VTE risk evaluation and VTE prophylaxis, and also the Padua scoring system through lectures, brochures and case-based learning. We did not use the latest tenth edition of the ACCP guidelines as it did not have updated information on prophylaxis for VTE. The effect of educational intervention was evaluated based on whether the project increased physician awareness of pharmacological thromboprophylaxis and the probability of VTE prevention. The study was approved by Shanghai Jiao Tong University School of Medicine, Ruijin Hospital ethics committee (approval No. 2018-59). Informed consent from participants was exempted by the ethics committee. All procedures performed in this study involving human participants were in accordance with the Declaration of Helsinki (as revised in 2013).

Inpatients from the respiratory department between February 2014 to December 2016, who were aged 18 years or older were included in our study. Patients were excluded if they were pregnant, with incomplete electronic medical records (including diagnostic records, instructions and other relevant information), hospitalized less than 2 days or had a VTE event on admission.

Since the QI project was started in April 2015, patients treated between April 2015 and December 2016 were assigned to the exposure or physician educational intervention (PEI) group, as their physicians had received the VTE prophylaxis educational intervention described above. Those who were treated between February 2014 and 


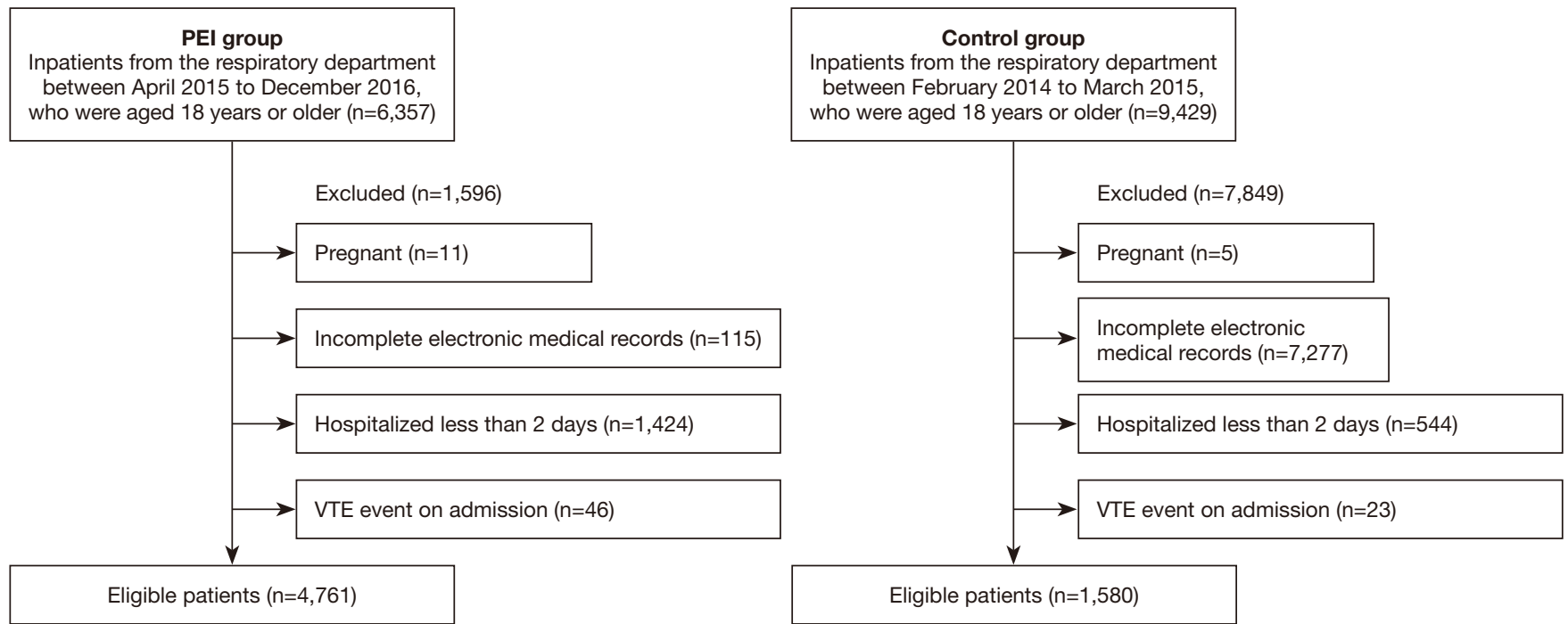

Figure 1 Flow chart of patient inclusion and exclusion criteria. PEI, physician educational intervention; VTE, venous thromboembolism.

March 2015 were assigned to the control group, as the QI project had not yet commenced during this period.

\section{Outcome assessment}

To determine the effect of educational intervention on physicians' awareness of pharmacological VTE prophylaxis, we calculated and compared any pharmacological VTE prophylaxis rates in overall patients, high VTE risk patients, high VTE risk patients with or without high major bleeding risk and low VTE risk patients in the two groups, and also appropriate pharmacological VTE prophylaxis rates in high VTE risk patients without high major bleeding risk. Any pharmacological VTE prophylaxis was defined as using any kinds of anticoagulants for medically ill patients with or without high VTE risk. Appropriate pharmacological VTE prophylaxis was defined as using LMWH, UFH or fondaparinux as pharmacological VTE prophylaxis for medically ill patients with high VTE risk and without high major bleeding risk, according to the ACCP guidelines (15-17). VTE risk was assessed using the Padua scoring system (18). Furthermore, we calculated and compared the use of appropriate anticoagulants as a proportion of all anticoagulants used in overall patients and high VTE risk patients. The three types of drugs mentioned above were regarded as appropriate anticoagulants. The VTE event occurrences in the two groups were also compared, and the relative risk (RR) of PEI on V'TE event occurrence was evaluated.

\section{Statistical analysis}

For baseline differences, demographic characteristics and duration of hospitalization were analyzed descriptively. The Wilcoxon-Mann-Whitney test was used for comparisons of age, body mass index (BMI) and duration of hospitalization between the study groups, as these measurement data did not conform to a normal distribution. A chi-squared test was used to compare differences in sex, VTE risk level and the distribution of VTE risk factor and co-morbidities between patients from the two groups, and also high major bleeding risk in high-risk patients. A chi-squared test and continuity correction were also used to compare the differences in any and appropriate pharmacological VTE prophylaxis rates, the proportion of appropriate anticoagulants out of all anticoagulants used, and the occurrence of VTE between the two groups. Poisson regression analysis was used to evaluate the RR of PEI on the occurrence of VTE events. VTE risk level, sex, duration of hospitalization (whether over 7 days), and the presence or absence of cancer were chosen as confounding factors. $\mathrm{P}$ values of $<0.05$ were considered statistically significant. We used SPSS Version 22.0 (IBM Corp., USA) to perform the statistical analyses.

\section{Results}

\section{Patient characteristics}

In total, our study involved 6,341 inpatients from the respiratory department (Figure 1). Of these patients, 4,761 
Table 1 Characteristics of the patients

\begin{tabular}{lcc}
\hline Variable & PEl group $(\mathrm{N}=4,761)$ & Control group $(\mathrm{N}=1,580)$ \\
\hline Sex & & $\mathrm{P}$ value \\
Female & $37.4 \%$ & $34.6 \%$ \\
Male & $62.6 \%$ & $65.4 \%$ \\
Age (years): median [IQR] & $61.25[15]$ & $60.48[16]$ \\
BMI (kg/m²): median [IQR] & $22.66[4.31]$ & $22.84[4.27]$ \\
Duration of hospitalization (days): median [IQR] & $6[6]$ & $6[7]$ \\
VTE risk level & & 0.015 \\
High-risk patients & $20.8 \%$ & $19.6 \%$ \\
Low-risk patients & $79.2 \%$ & $80.4 \%$ \\
\hline
\end{tabular}

BMI, body mass index; IQR, interquartile range; PEI, physician educational intervention; SD, standard deviation; VTE, venous thromboembolism.

Table 2 Co-morbidities of the patients

\begin{tabular}{|c|c|c|c|}
\hline Co-morbidities & PEI group $(\mathrm{N}=4,761)(\%)$ & Control group $(\mathrm{N}=1,580)(\%)$ & $P$ value \\
\hline Severe respiratory disease & 13.1 & 14.7 & 0.107 \\
\hline Heart failure & 2.5 & 3.2 & 0.128 \\
\hline Acute stroke & 2.1 & 4.0 & $<0.001$ \\
\hline Rheumatic diseases & 1.8 & 1.4 & 0.318 \\
\hline
\end{tabular}

PEI, physician educational intervention.

were assigned to the PEI group and 1,580 patients were assigned to the control group (Table 1). Patients in the PEI group [median 61.25 (IQR 15) years] were older than those in the control group [median 60.48 (IQR 16) years] $(\mathrm{P}=0.015)$. Compared with the control group, the proportion of female patients was higher $(37.4 \%$ vs. $34.6 \%, \mathrm{P}=0.040)$ and the duration of hospitalization was shorter [median 6 (IQR 6) vs. median 6 (IQR 7) days, $\mathrm{P}<0.001]$ in the PEI group. There was no significant difference in BMI between the two groups [PEI group: median 22.66 (IQR 4.31), control group: median 22.84 (IQR 4.27), $\mathrm{P}=0.287$ ]. Major co-morbidities observed in patients included active cancer, severe respiratory disease, heart failure, acute stroke, acute infection and rheumatic diseases (Table 2).

High-risk patients took up $20.8 \%$ of the PEI group and $19.6 \%$ of the control group, respectively $(\mathrm{P}=0.291)$. The major risk factors of the PEI group were active cancer $(73.9 \%)$, elderly age ( $\geq 70$ years) $(24.2 \%)$, heart and/or respiratory failure (6.9\%). Active cancer (67.3\%), elderly age ( $\geq 70$ years) $(22.7 \%)$, acute infection and/or rheumatologic disorder $(7.0 \%)$ were major risk factors for the control group (Table 3). Of the high-risk patients, $42.0 \%$ in the PEI group and $50.5 \%$ in the control group were with high major bleeding risk $(\mathrm{P}=0.009)$.

\section{Outcomes}

\section{Pharmacological VTE prophylaxis rates}

A total of $5.6 \%$ and $4.7 \%$ of overall patients in the PEI group and control group received any pharmacological VTE prophylaxis, respectively. In high VTE risk patients, $10 \%$ of these patients in the PEI group received any pharmacological VTE prophylaxis, compared with $7.4 \%$ in the control group. The difference was not statistically significant $(\mathrm{P}=0.179) .11 .3 \%$ and $5.9 \%$ of high VTE risk patients without high major bleeding risk in the PEI group 
Table 3 Distribution of VTE risks

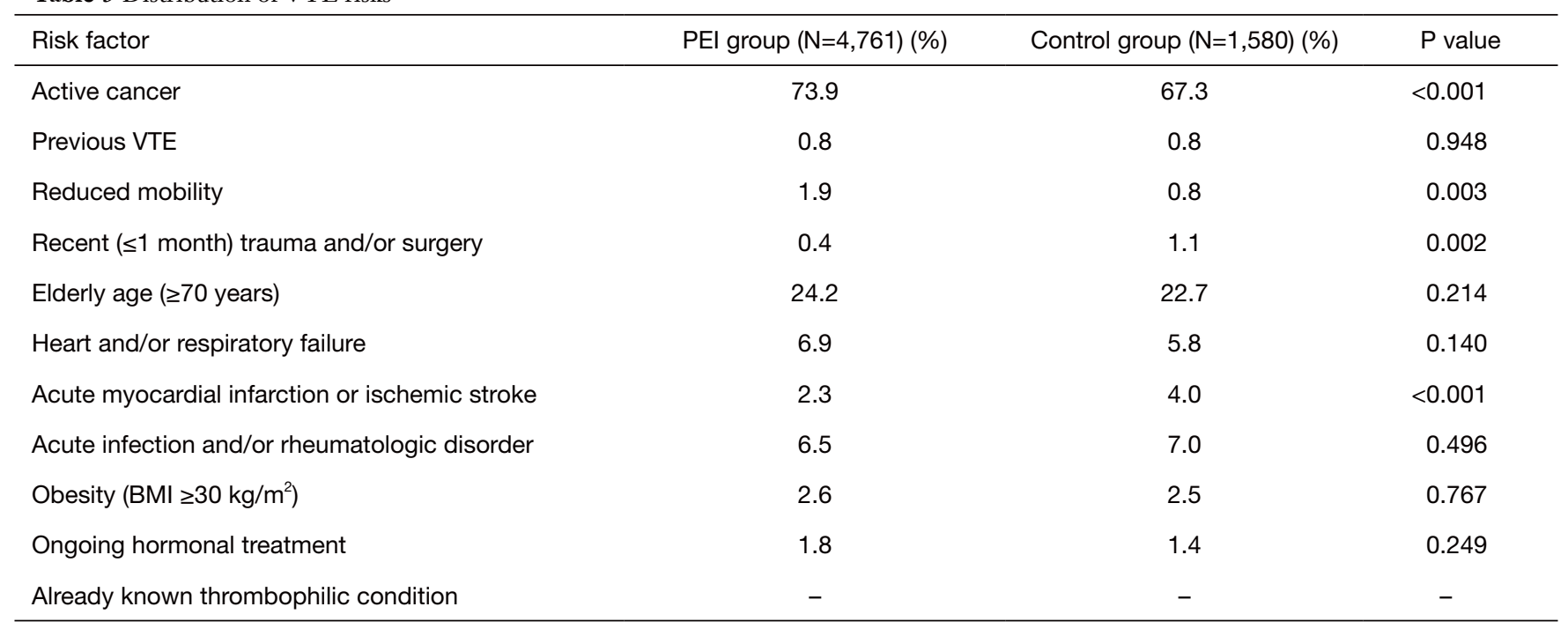

VTE, venous thromboembolism; BMI, body mass index; PEI, physician educational intervention.
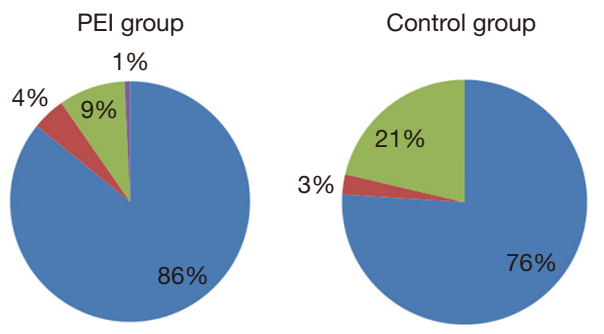

- LMWH
UFH
- Aspirin
a Warfarin

Figure 2 Anticoagulants used in the study. LMWH was the most used anticoagulant across the two groups. LMWH, low-molecularweight heparin; PEI, physician educational intervention; UFH, low-dose unfractionated heparin.

and control group received any pharmacological VTE prophylaxis, respectively $(\mathrm{P}=0.048)$. There were also $8.2 \%$ of high VTE risk patients with high major bleeding risk in the PEI group and $9.0 \%$ in the control group received any pharmacological VTE prophylaxis $(\mathrm{P}=0.758)$. And the appropriate pharmacological VTE prophylaxis rate was significantly higher in the PEI group $(10.6 \%)$ than the control group (4.6\%) in high VTE risk patients without high major bleeding risk $(\mathrm{P}=0.022)$, who should receive VTE prophylaxis according to the ACCP guidelines. As for low VTE risk patients, any pharmacological VTE prophylaxis rates were similar between the two groups (PEI group: $4.5 \%$, control group: $4.1 \%, \mathrm{P}=0.557$ ).

\section{The use of anticoagulants}

We recorded the exact anticoagulants patients were treated with, and found that LMWH was the most used anticoagulant in both the PEI group $(85.8 \%)$ and the control group $(76.0 \%)(\mathrm{P}=0.042)$. Other anticoagulants used in the present study included UFH, aspirin and warfarin (Figure 2). Among them, LMWH and UFH were appropriate anticoagulants according to the ACCP guidelines. In the PEI group, appropriate anticoagulants comprised $90.3 \%$ of all used anticoagulants, which was higher than that of control group $(78.7 \%)(\mathrm{P}=0.007)$. Moreover, for anticoagulants used in high-risk patients without high major bleeding risk, appropriate anticoagulants were at a proportion of $93.8 \%$ in the PEI group and $77.8 \%$ in the control group $(\mathrm{P}=0.153)$. Among them, $\mathrm{LMWH}$ took up $95.1 \%$ and $100 \%$ in the PEI group and control group respectively $(\mathrm{P}=1.000)$.

\section{The occurrence of VTE}

There were 26 VTE events in the PEI group, including 17 PE events, 8 DVT events and 1 DVT combined with a PE event. Of these, $61.5 \%$ were classified as high VTE risk patients. In the control group, 9 VTE events occurred, including 3 PE events and 6 DVT events. Of these, 33.3\% were classified as high VTE risk patients (Table 4). The likelihood of VTE event occurrence was similar between the two groups (PEI group: 0.5\%, control group: 0.6\%, 
Table 4 VTE event occurrences according to patient risk in the PEI group and the control group

\begin{tabular}{lcc}
\hline VTE events & PEl group (N=26) (\%) & Control group (N=9) (\%) \\
\hline PE only & & 0.0 \\
High VTE risk patients & 42.3 & 33.3 \\
Low VTE risk patients & 23.1 & 33.3 \\
DVT only & 19.2 & 33.3 \\
High VTE risk patients & 11.5 & 0.0 \\
Low VTE risk patients & & 0.0 \\
PE \& DVT & 0.0 & 3.8 \\
High VTE risk patients & & \\
Low VTE risk patients & & \\
\hline
\end{tabular}

VTE, venous thromboembolism; DVT, deep vein thrombosis; PE, pulmonary embolism; PEI, physician educational intervention.

$\mathrm{P}=0.913$ ), and between the subgroups of patients with high VTE risk (PEI group: 1.7\%, control group: $1.0 \%, \mathrm{P}=0.554$ ). We also noted that 4 VTE events in the PEI group were asymptomatic, accounting for $15.4 \%$ of all VTE events in this group, while no asymptomatic VTE events were identified in the control group.

We performed Poisson regression analysis to evaluate the effect of PEI on the occurrence of VTE events after controlling for potential confounding factors (VTE risk level, sex, duration of hospitalization, presence/absence of cancer). Results indicated that PEI was not associated with the probability of VTE event occurrence (RR, 1.246; 95\% CI, 0.478-2.188, $\mathrm{P}=0.954)$. In addition, Poisson regression analysis also showed that high VTE risk (RR, 0.229; 95\% CI, 0.111-0.473; $\mathrm{P}<0.001)$, duration of hospitalization over 7 days $(\mathrm{RR}, 0.271 ; 95 \% \mathrm{CI}, 0.130-0.5634 ; \mathrm{P}<0.001)$ and male gender (RR, 0.480; 95\% CI, 0.246-0.937, $\mathrm{P}=0.031$ ) were risk factors for VTE events.

\section{Discussion}

The present study demonstrated that PEI for VTE prophylaxis effectively increased any and appropriate pharmacological VTE prophylaxis rates in high-risk patients without high major bleeding risk, as well as the use of appropriate anticoagulants, though failed to decrease VTE event occurrence. This is the first real-world study focusing on the effectiveness of educational intervention on VTE prophylaxis in a Chinese population. The study also showed the effect of PEI on the occurrence of VTE events, as most previous studies are lacking in the relevant data.
Our results provide significant evidence on how to improve VTE prophylaxis in medical inpatients at the physician, hospital administration and government level.

Several studies have reported the effect of educational intervention on promoting physician awareness of VTE prophylaxis. Ongen et al. found that physician training significantly increased VTE prophylaxis rates from $49.4 \%$ to $62.4 \%$ in patients, and led to a higher rate of VTE risk evaluation (11). Furthermore, a study by Al-Hameed et al. showed that educational intervention increased VTE prophylaxis utilization from $36.5 \%$ to $63.9 \%$ in VTE patients (13). In the present study, for high-risk patients without high major bleeding risk, any and appropriate pharmacological VTE prophylaxis rates were all higher after educational intervention. Also, appropriate anticoagulants made up a larger proportion of all used anticoagulants. Thus, our results indicate that QI projects can effectively increase physician awareness of pharmacological thromboprophylaxis through educational intervention.

A previous study established that PEI effectively decreased VTE incidence up to 51\% (19). However, in our study, the occurrence of VTE events did not decrease after PEI, even with increased appropriate pharmacological VTE prophylaxis rates. Poisson regression analysis also showed that PEI had no association with the probability of VTE event occurrence. This may be due to an insufficient increase in patients receiving pharmacological VTE prophylaxis, a low incidence of VTE, and a small sample size. Furthermore, the absence of records for discharged patients in our study made analysis of VTE event occurrences between the two groups difficult, as 
most VTE events occur after discharge $(1,20)$. However, we found that nearly one-sixth of patients who experienced VTE events were asymptomatic in the PEI group, while no asymptomatic VTE events were observed in the control group. This indicates that with improved awareness of VTE management, physicians diagnosed patients earlier and the VTE detection rate increased, which therefore resulted in no decreases in VTE event occurrence after the QI project was carried out.

We also found that the guidelines were implemented to a relatively low degree in this study. In both the PEI group and the control group, any and appropriate pharmacological VTE prophylaxis rates were extremely low in high-risk patients, who should take pharmacological thromboprophylaxis according to the ACCP guidelines (15-17). A nationwide, multicenter, cross-sectional study of VTE prophylaxis in Chinese inpatients demonstrated any and appropriate VTE prophylaxis rates of $12.9 \%$ and $6.0 \%$ in medical inpatients with high VTE risk, which were relatively consistent with our results (21). However, studies from Western countries, such as ENDORSE and VOICE, have demonstrated higher appropriate pharmacological VTE prophylaxis rates at approximately $16-50.2 \%$ for inpatients $(10,22,23)$. This reflects a lack of awareness of VTE prophylaxis in Chinese physicians. Furthermore, we found pharmacological VTE prophylaxis in low-risk patients and high-risk patients with high major bleeding risk, indicating an overuse of pharmacological prophylaxis in these patients, for whom pharmacological prophylaxis are not recommended according to the ACCP guidelines. There were also inappropriate usages of anticoagulants. Aspirin and warfarin were wrongly used as anticoagulants in the present study, with fondaparinux not being used. Although not mentioned in our results, the dose and duration of pharmacological VTE prophylaxis can also be inappropriate (24). The causes of non-compliance with guidelines are often complicated, and can include insufficient education of VTE prophylaxis, and a lack of measures made by hospital management and the government (21).

To fill the gaps between the guidelines and clinical practice, repeated physician education intervention by multiple media (e.g., lecture, brochure, video, slides and application in cell phone) and exams can be adapted to strengthen the education intervention. Further interventions such as uniform VTE and bleeding risk assessment, green channel, multidisciplinary teams, patient education of VTE and discharged patient follow-up system are also necessary.

In addition to high VTE risk, we also found duration of hospitalization over 7 days was a strong risk factor for VTE. Amin et al. found that with longer hospital stays, VTE occurrence increased from $0.5 \%$ to $5.4 \%$ (25). Holmqvist $e t$ al. also found that hospitalization was a risk factor for VTE the first year after discharge in patients with rheumatoid arthritis (26). This may be due to patients getting less exercise and having more time lying in bed during hospitalization, as reduced mobility is one of the risk factors for VTE according to the Padua scoring system (18). Thus, reducing hospitalization length may also be helpful for VTE prevention.

Since most of the data on VTE comes from randomized clinical trials, for which almost one-quarter of VTE patients meet at least 1 exclusion criteria and are therefore ineligible, there is a lack of data on real-world management of VTE (3). The present retrospective cohort study involving 6,341 patients may provide effective supporting evidence. Furthermore, our study also provides insights in an area with a scarcity of information, namely, educational intervention for VTE prophylaxis in a Chinese population. We evaluated any and appropriate pharmacological VTE prophylaxis rates, the use of anticoagulants, and the occurrence of VTE events before and after carrying out PEI for VTE prophylaxis in the respiratory department. We believe our results are of great significance not just for physicians, but also hospital management and government policy.

This study has several limitations. First, it is a singlecenter retrospective observational cohort study. Thus, some important confounding factors might not have been controlled when determine the causal inferences between physicians education intervention and appropriate pharmacological VTE prophylaxis. And the results might be limited in terms of generalizability across the population. We have used large sample size and strict inclusion and exclusion criteria to decrease the deviation of the study. Second, this study only focused on the respiratory department, therefore it is lacking data on medical inpatients from other departments and investigations on other physicians who received educational intervention. Furthermore, baseline data were unbalanced between the two groups, which might have introduced bias in our results. The unbalanced baseline may due to the limitation of the retrospective single-center real world study design. Besides, the shortening duration of hospitalization, increasing aging population and average life span in recent years can also cause unbalanced baseline. Finally, the present study involving inpatients did not focus on the long-term effects 
of VTE prophylaxis, however, as noted above, most VTE events occur in discharged patients.

\section{Conclusions}

In conclusion, our results demonstrated that PEI effectively increased physician awareness of VTE prophylaxis, as it significantly raised the any and appropriate pharmacological VTE prophylaxis rates in high-risk patients without high major bleeding risk and the use of appropriate anticoagulants. Further interventions are still necessary as the guidelines were implemented to a relatively low degree.

\section{Acknowledgments}

Funding: This work was financially supported by the Action Plan of Major Diseases Prevention and Treatment (2017ZX01001-S12), the Project of Integrating Chinese and Western Medicine in General Hospital (ZHYYZXYJHZX-201901) and the Project of Continuous Improvement of Medical Quality Evidence-based Management (YLZLXZ-2020-008).

\section{Footnote}

Reporting Checklist: The authors have completed the STROBE reporting checklist. Available at http://dx.doi. org/10.21037/apm-20-1833

Data Sharing Statement: Available at http://dx.doi. org/10.21037/apm-20-1833

Conflicts of Interest: All authors have completed the ICMJE uniform disclosure form (available at http://dx.doi. org/10.21037/apm-20-1833). HW reports grants from Development Center for Medical Science \& Technology National Health Commission of the People's Republic of China, grants from Shanghai Municipal Health Commission, Shanghai Municipal Administrator of Traditional Chinese Medicine, grants from National Institute of Hospital Administration of the People's Republic of China, during the conduct of the study; YP reports grants from Development Center for Medical Science \& Technology National Health Commission of the People's Republic of China, grants from Shanghai Municipal Health Commission, Shanghai Municipal Administrator of Traditional Chinese Medicine, grants from National Institute of Hospital Administration of the People's Republic of China, during the conduct of the study; TC reports grants from Development Center for Medical Science \& Technology National Health Commission of the People's Republic of China, grants from Shanghai Municipal Health Commission, Shanghai Municipal Administrator of Traditional Chinese Medicine, grants from National Institute of Hospital Administration of the People's Republic of China, during the conduct of the study; LC reports grants from Development Center for Medical Science \& Technology National Health Commission of the People's Republic of China, grants from Shanghai Municipal Health Commission, Shanghai Municipal Administrator of Traditional Chinese Medicine, grants from National Institute of Hospital Administration of the People's Republic of China, during the conduct of the study; HL reports grants from Development Center for Medical Science \& Technology National Health Commission of the People's Republic of China, grants from Shanghai Municipal Health Commission, Shanghai Municipal Administrator of Traditional Chinese Medicine, grants from National Institute of Hospital Administration of the People's Republic of China, during the conduct of the study; EC reports grants from Development Center for Medical Science \& Technology National Health Commission of the People's Republic of China, grants from Shanghai Municipal Health Commission, Shanghai Municipal Administrator of Traditional Chinese Medicine, grants from National Institute of Hospital Administration of the People's Republic of China, during the conduct of the study; JQ reports grants from Development Center for Medical Science \& Technology National Health Commission of the People's Republic of China, grants from Shanghai Municipal Health Commission, Shanghai Municipal Administrator of Traditional Chinese Medicine, grants from National Institute of Hospital Administration of the People's Republic of China, during the conduct of the study; WT reports grants from Development Center for Medical Science \& Technology National Health Commission of the People's Republic of China, grants from Shanghai Municipal Health Commission, Shanghai Municipal Administrator of Traditional Chinese Medicine, grants from National Institute of Hospital Administration of the People's Republic of China, during the conduct of the study; WX reports grants from Development Center for Medical Science \& Technology National Health Commission of the People's Republic of China, grants from Shanghai Municipal Health Commission, Shanghai Municipal Administrator of Traditional Chinese Medicine, 
grants from National Institute of Hospital Administration of the People's Republic of China, during the conduct of the study; YL reports grants from Development Center for Medical Science \& Technology National Health Commission of the People's Republic of China, grants from Shanghai Municipal Health Commission, Shanghai Municipal Administrator of Traditional Chinese Medicine, grants from National Institute of Hospital Administration of the People's Republic of China, during the conduct of the study. The authors have no other conflicts of interest to declare.

Ethical Statement: The authors are accountable for all aspects of the work in ensuring that questions related to the accuracy or integrity of any part of the work are appropriately investigated and resolved. All procedures performed in this study involving human participants were in accordance with the Declaration of Helsinki (as revised in 2013). The study was approved by Shanghai Jiao Tong University School of Medicine, Ruijin Hospital ethics committee (approval No. 2018-59). Informed consent from participants was exempted by the ethics committee.

Open Access Statement: This is an Open Access article distributed in accordance with the Creative Commons Attribution-NonCommercial-NoDerivs 4.0 International License (CC BY-NC-ND 4.0), which permits the noncommercial replication and distribution of the article with the strict proviso that no changes or edits are made and the original work is properly cited (including links to both the formal publication through the relevant DOI and the license). See: https://creativecommons.org/licenses/by-nc-nd/4.0/.

\section{References}

1. Ungprasert P, Crowson CS, Matteson EL. Association of sarcoidosis with increased risk of VTE: A PopulationBased Study, 1976 to 2013. Chest 2017;151:425-30.

2. Heit JA, Crusan DJ, Ashrani AA, et al. Effect of a nearuniversal hospitalization-based prophylaxis regimen on annual number of venous thromboembolism events in the US. Blood 2017;130:109-14.

3. Bahloul M, Dlela M, Khlaf Bouaziz N, et al. Early posttraumatic pulmonary-embolism in patients requiring ICU admission: more complicated than we think! J Thorac Dis 2018;10:S3850-4.

4. Yamashita Y, Morimoto T, Amano H, et al. Anticoagulation Therapy for Venous Thromboembolism in the Real World- From the COMMAND VTE Registry. Circ J 2018;82:1262-70.

5. Kawaler E, Cobian A, Peissig P, et al. Learning to predict post-hospitalization VTE risk from EHR data. AMIA Annu Symp Proc 2012;2012:436-45.

6. Khalafallah AA, Kirkby BE, Wong S, et al. Venous thromboembolism in medical patients during hospitalisation and 3 months after hospitalisation: a prospective observational study. BMJ Open 2016;6:e012346.

7. Aissa I, Rachdi I, Ben Miled K, et al. Incidence of venous thromboembolism in men admitted to a pneumology unit for acute respiratory disease. Rev Pneumol Clin 2011;67:129-35.

8. Cohen AT, Nandini B, Wills JO, et al. VTE prophylaxis for the medical patient: where do we stand? - A focus on cancer patients. Thromb Res 2010;125 Suppl 2:S21-9.

9. Hemon F, Fouchard F, Tromeur C, et al. Association between hospitalization for acute medical illness and VTE risk: A lower efficacy of thromboprophylaxis in elderly patients? Results from the EDITH case-control study. Eur J Intern Med 2017;44:39-43.

10. Cohen AT, Tapson VF, Bergmann JF, et al. Venous thromboembolism risk and prophylaxis in the acute hospital care setting (ENDORSE study): a multinational cross-sectional study. Lancet 2008;371:387-94.

11. Ongen G, Demir M, Molinas N, et al. Evaluation of the Practice Pattern of Medical Patients' VTE Prophylaxis With a Standard Risk Assessment Model Form: MERAM Study. Clin Appl Thromb Hemost 2015;21:412-9.

12. Myers MK, Jansson-Knodell CL, Schroeder DR, et al. Using knowledge translation for quality improvement: an interprofessional education intervention to improve thromboprophylaxis among medical inpatients. J Multidiscip Healthc 2018;11:467-72.

13. Al-Hameed F, Al-Dorzi HM, Aboelnazer E. The effect of a continuing medical education program on Venous thromboembolism prophylaxis utilization and mortality in a tertiary-care hospital. Thromb J 2014;12:9.

14. Leong L, Mendelsohn M, Saavedra C, et al. Quality improvement education for venous thromboembolism (VTE) prevention in cancer. J Contin Educ Health Prof 2015;35 Suppl 1:S29-30.

15. Guyatt GH, Eikelboom JW, Gould MK, et al. Approach to outcome measurement in the prevention of thrombosis in surgical and medical patients: Antithrombotic Therapy and Prevention of Thrombosis, 9th ed: American College of Chest Physicians Evidence-Based Clinical Practice 
Guidelines. Chest 2012;141:e185S-94S.

16. Kahn SR, Lim W, Dunn AS, et al. Prevention of VTE in nonsurgical patients: Antithrombotic Therapy and Prevention of Thrombosis, 9th ed: American College of Chest Physicians Evidence-Based Clinical Practice Guidelines. Chest 2012;141:e195S-e226S.

17. Lansberg MG, O’Donnell MJ, Khatri P, et al. Antithrombotic and thrombolytic therapy for ischemic stroke: Antithrombotic Therapy and Prevention of Thrombosis, 9th ed: American College of Chest Physicians Evidence-Based Clinical Practice Guidelines. Chest 2012;141:e601S-36S

18. Barbar S, Noventa F, Rossetto V, et al. A risk assessment model for the identification of hospitalized medical patients at risk for venous thromboembolism: the Padua Prediction Score. J Thromb Haemost 2010;8:2450-7.

19. Pingleton SK, Carlton E, Wilkinson S, et al. Reduction of Venous Thromboembolism (VTE) in Hospitalized Patients: Aligning Continuing Education With Interprofessional Team-Based Quality Improvement in an Academic Medical Center. Acad Med 2013;88:1454-9.

20. Kukreja JE, Levey HR, Scosyrev E, et al. Effectiveness and safety of extended-duration prophylaxis for venous thromboembolism in major urologic oncology surgery. Urol Oncol 2015;33:387.e7-16.

21. Zhai Z, Kan Q, Li W, et al. VTE Risk Profiles and Prophylaxis in Medical and Surgical Inpatients: The

Cite this article as: Wang $\mathrm{H}$, Pan Y, Chen T, Chen L, Lv H, Chen E, Qu J, Tang W, Xu W, Lu Y. The effect of physician educational intervention on venous thromboembolism pharmacological prophylaxis in medical inpatients from the respiratory department: a retrospective cohort study. Ann Palliat Med 2020;9(6):3966-3975. doi: 10.21037/apm-20-1833
Identification of Chinese Hospitalized Patients' Risk Profile for Venous Thromboembolism (DissolVE-2)-A Cross-sectional Study. Chest 2019;155:114-22.

22. Cionac Florescu S, Anastase DM, et al. Venous thromboembolism following major orthopedic surgery. Maedica. 2013;8:189-94.

23. Kahn SR, Panju A, Geerts W, et al. Multicenter evaluation of the use of venous thromboembolism prophylaxis in acutely ill medical patients in Canada. Thromb Res 2007;119:145-55.

24. Amin A, Spyropoulos AC, Dobesh P, et al. Are hospitals delivering appropriate VTE prevention? The venous thromboembolism study to assess the rate of thromboprophylaxis (VTE start). J Thromb Thrombolysis 2010;29:326-39.

25. Amin A, Neuman WR, Lingohr-Smith $M$, et al. Influence of the duration of hospital length of stay on frequency of prophylaxis and risk for venous thromboembolism among patients hospitalized for acute medical illnesses in the USA. Drugs Context 2019;8:212568.

26. Holmqvist ME, Neovius M, Eriksson J, et al. Risk of venous thromboembolism in patients with rheumatoid arthritis and association with disease duration and hospitalization. JAMA 2012;308:1350-6.

(English Language Editor: C. Betlazar-Maseh) 